\title{
Fonction publique territoriale : de la mobilité interne à la mobilité spatiale?
}

Civil servants and geographical mobility in the XIX ${ }^{\text {th }}$ century. The case of the tax collectors

\section{Émilie Biland}

\section{(2) OpenEdition}

\section{Journals}

Édition électronique

URL : http://journals.openedition.org/travailemploi/5328

DOI : 10.4000/travailemploi.5328

ISSN : $1775-416 \mathrm{X}$

Éditeur

DARES - Ministère du Travail

Édition imprimée

Date de publication : 15 septembre 2011

Pagination : 53-66

ISSN : 0224-4365

Référence électronique

Émilie Biland, «Fonction publique territoriale : de la mobilité interne à la mobilité spatiale? », Travail et Emploi [En ligne], 127 | juillet-septembre 2011, mis en ligne le 25 février 2014, consulté le 19 avril 2019. URL : http://journals.openedition.org/travailemploi/5328; DOI : 10.4000/travailemploi.5328 


\title{
Fonction publique territoriale : de la mobilité interne à la mobilité spatiale?
}

\author{
Émilie Biland (*)
}

\begin{abstract}
Au contraire de leurs homologues de l'État, les trajectoires des agents des collectivités territoriales ont longtemps été marquées par la stabilité, plutôt que par la mobilité spatiale. Cet article revient sur ce modèle de recrutement et de carrière au local et analyse sa remise en cause contemporaine, au moyen d'une analyse quantitative et qualitative de dossiers du personnel d'une petite ville. La montée en puissance de la mobilité géographique est concomitante de la raréfaction de la mobilité sociale en cours de vie active. Toutefois, la majorité des agents publics de cette ville demeurent ancrés localement. Pour comprendre la percée limitée de cette mise en mobilité des carrières professionnelles, l'article repère ses coûts et bénéfices inégaux selon des facteurs générationnels, sociaux et genrés.
\end{abstract}

«Si vous n'êtes pas nommés trois mois après avoir réussi le concours, la France vous tend les bras! Les concours ont valeur nationale et on ne pourra pas vous muter d'autorité comme dans la Fonction Publique de l'État. Et la Savoie est belle!»

Prononcés avec enthousiasme par un haut fonctionnaire du conseil général de Savoie lors du salon de l'emploi public organisé en $2007^{(1)}$, ces paroles sont emblématiques des encouragements contemporains à la mobilité spatiale dans la fonction publique territoriale (FPT). Selon les dires de son auteur, cette dernière donnerait accès à des changements vécus positivement, car choisis plutôt que contraints. Prenant ses distances avec la fonction publique de l'État (FPE) - selon une posture ancienne, et ravivée depuis la décentralisation - ce cadre territorial fait également la promotion de son département, rappelant que, pour le recrutement de leurs personnels comme pour attirer les entreprises, les collectivités ont pris la voie du «marketing territorial», notamment par l'octroi de primes (LE BART, 2011).

Cette prise de position invite à l'examen des différentes formes de mobilité dans l'emploi public local. Disons-le d'emblée, les discours du salon de l'emploi public prennent à contre-pied l'image - en grande partie fondée empiriquement, on le verra - d'une fonction publique historiquement peu mobile. L'existence de liens étroits entre les agents publics locaux et leur territoire de travail et de résidence constitue en effet un topos régulièrement utilisé pour décrire ce segment de l'emploi

(*) Université de Laval, Québec, Canada; emilie.biland@pol. ulaval.ca

(1) Agora, «Intégrer la fonction publique : comment préparer un concours?», $4^{\mathrm{e}}$ salon de l'emploi public, Palais des congrès de Paris, 29 mars 2007. public. À Tozay, la ville étudiée dans la suite de cet article, les trois-quarts des emplois municipaux sont occupés par des résidents. Plus de la moitié des agents sont même nés dans la ville et les trois-quarts dans le département.

En fait, la mobilité géographique, à la fois comme obligation statutaire, norme corporative et contrainte pratique, n'a jamais été présente dans la FPT comme elle l'a été dans la FPE, connue pour produire des «déracinés» (Blum, DE LA GoRCE, THÉLOT, 1985, p. 424). Les historiens de la Troisième République ont bien montré que l'appartenance locale fonctionnait à l'inverse comme un critère de priorité à l'embauche dans les collectivités. Le recrutement de nouveaux venus (provinciaux) n'était certes pas impossible, en région parisienne en particulier, mais il stabilisait leurs trajectoires résidentielles et participait à leur intégration aux sociétés banlieusardes (BELlanger, 2008). Ayant persisté, quoiqu'à des degrés divers selon les employeurs et les catégories hiérarchiques jusqu'aux années 1970 voire 1980, ce modèle a depuis été battu en brèche. Au moment où le nouvel esprit du capitalisme valorise la flexibilité contre la rigidité et l'adaptabilité contre l'enracinement local (Boltanski, ChIAPELlo, 1999, p. 179), l'emploi public local serait-il à son tour affecté par la «mise en mobilité généralisée» des carrières professionnelles (CASTEL, 2003, p. 43) dont les enquêtes Emploi et $F Q P$ ont bien montré l'importance dans la période postfordiste (CHAPOULIE, 2000)?

Pour répondre à cette question, cet article examine les discours et les textes juridiques relatifs à la FPT, devenus favorables à la mobilité géographique, aux formes effectives de mobilité des agents publics locaux. On testera ici l'hypothèse du déclin de la mobilité professionnelle interne 
à la collectivité employeuse au profit de la mobilité géographique, elle-même support d'ascension professionnelle. Les conditions de mobilité sociale par le travail ${ }^{(2)}$ se seraient donc transformées depuis les années 1970? La fonction publique fut, dans les quatre décennies d'après-guerre, un «milieu particulièrement favorable à l'ascension sociale» (Gollac, 2005, pp. 50-51), en particulier pour les enfants d'ouvriers et les femmes. Dans des collectivités dont les agents sont moins diplômés que ceux de l'État, les trajectoires d'ascension sociale intergénérationnelle ne s'expliquaient pas tant par la réussite scolaire que par le mode de production interne de l'encadrement, selon une logique «localiste-municipaliste» (Burlen, Thoenig, 1998). Cette association du recrutement local et de la mobilité interne est aujourd'hui remise en cause par l'élévation de la sélectivité scolaire à l'embauche et par le développement des mutations entre collectivités. La mobilité horizontale, entre fonctions comme entre employeurs, ferait désormais partie des conditions - aux côtés de la détention de diplômes - à la mobilité verticale, selon la distinction introduite par Howard BECKER (1970, pp. 165-176).

Pour examiner l'articulation entre ces deux types de mobilité dans la fonction publique territoriale, l'article associe des matériaux qualitatifs et quantitatifs. Dans la première partie, il approche «par le haut» l'inflexion en faveur de la mobilité spatiale intervenue depuis une trentaine d'années. Analysant les discours tenus par les responsables politiques et administratifs de ce segment de la fonction publique ainsi que par ses analystes, il montre que les transformations juridiques et morphologiques de la FPT soutiennent leurs aspirations. La deuxième partie précise ces constats au moyen d'une enquête localisée dans une petite ville de province. L'examen quantitatif des mobilités connues par ses agents permet de saisir la structuration genrée, sociale et générationnelle des carrières professionnelles passées des agents. Loin des inflexions observées dans le sens d'une montée de la mobilité géographique, la réalité d'une administration municipale de taille moyenne demeure très majoritairement celle d'agents du cru. Complétée par la présentation de parcours effectifs d'agents publics, cette approche «par le bas » débouche sur l'identification de formes typiques de mobilités, rendant compte de l'hétérogénéité de cette fonction publique.

\section{Des textes et des discours favorables à la mobilité spatiale}

\section{De la valorisation à la critique des fonctionnaires "du cru "}

Reflet de l'émiettement communal français, l'emploi public local a longtemps été un «continent morcelé» (Bellanger, 2004, p. 294). Ce morcèlement était sensible au sein de chaque administration communale, du fait de la diversité des milieux sociaux représentés et des emplois occupés, mais plus encore entre les agents des différentes communes. En effet, jusqu'en 1972, il n'était pas possible de changer de mairie sans perdre son ancienneté et aucun mécanisme de mise en publicité des postes vacants (ce que l'on appelle aujourd'hui les bourses d'emploi) ne fonctionnait véritablement. De surcroît, en vertu du statut du personnel communal de 1952(3), les employeurs avaient la possibilité de déroger au tableau des emplois fixé par le ministère de l'Intérieur en créant des emplois spécifiques, dont l'occupation rendait encore plus délicate le changement d'employeur. Précocement raillé sous les traits du clochemerlisme (CHEVALLIER, 1934), l'attachement des employés communaux à leur territoire de travail, qui était souvent également leur lieu de vie, n'en a pas moins constitué la norme dans les deux décennies d'après-guerre ${ }^{(4)}$. Au début des années 1970, lors des débats préalables à la loi rendant possible les mutations ${ }^{(5)}$, les députés et sénateurs-maires les plus ancrés localement insistent encore sur l'«intimité entre mairie et société locale» (LoRRAIN, 1991, p. 467) et sur les liens quasi-paternels qui uniraient les maires à leurs personnels. Maujoüan, député républicain-indépendant, parle des «qualités humaines, de jugement et sérieux», qui permettent au secrétaire de mairie d'être le "confident» des habitants, tandis que le sénateur socialiste Nayrou se souvient d'un agriculteur, secrétaire de mairie avant la guerre, dont l'activité professionnelle et le «dévouement» assuraient «l'intégration au milieu».

Mais ces débats attestent également de l'émergence d'une nouvelle représentation de l'emploi communal. Depuis la fin des années 1960, s'organise en effet un mouvement réformateur, porté par des hommes politiques à la carrière moins locale,

(3) Loi du 28 avril 1952

(4) Les zones les plus urbaines, telle que la petite couronne parisienne, font à cet égard figure d'exception : des dispositifs de mise en circulation des personnels - tels que les concours intercommunaux $-\mathrm{y}$ ont été mis en place dans l'entre-deuxguerres (BELlanger, 2001).

(5) Et inaugurant, surtout, une politique de formation continue pour les agents publics locaux. Ces débats peuvent être consultés dans Journal Officiel, 1971, Sénat, Débats Parlementaires, 2. session 1970-1971, janvier-mai 1971, p. 243-264; Journal Officiel, Assemblée Nationale, Débats Parlementaires, 2e session, 1971-1972, 22-35, mai 1972, pp. 1998-2011.
(2) Ce texte traite principalement de la promotion professionnelle en cours de vie active, les données quantitatives manquant pour estimer l'ampleur de la mobilité sociale intergénérationnelle. 
d'universitaires (surtout juristes) et des fonctionnaires de l'État, qui remet progressivement en cause «l'immobilisme» des agents publics locaux. Le sénateur centriste Schiélé vante par exemple les concours intercommunaux, qui «libèrent [le maire] des servitudes attachées au recrutement sur place, lui donne le recul et l'indépendance qui conviennent pour échapper aux pesanteurs locales, parfois très gênantes ». Plus que l'insuffisante mobilité géographique, c'est le manque de qualification des personnels ${ }^{(6)}$ qui retient d'abord leur attention : pour des communes dotées de plus de pouvoirs, il faut des agents mieux formés. La loi du 13 juillet 1972 met ainsi en place le Centre de formation des personnels communaux, qui organise des concours internes, lesquels assoient les promotions, non seulement sur le repérage édilitaire, mais aussi sur les capacités.

Dans les années 1970 et 1980, les cadres communaux - soucieux de se distinguer de leurs homologues de l'État - continuent de revendiquer leur connaissance du «quotidien, du réel, du concret» (FonTAINe, 1986, p. 35) et des « besoins de la population» (LoRRAIN, 1991, p. 468). Mais plusieurs observateurs du monde communal critiquent leur «fonctionnement conservateur, attaché aux pratiques établies et réfractaires à la nouveauté» (Dion, 1986, p. 23), qu'ils imputent à la structure des carrières marquée par leur «aspect statutaire, leur absence de mobilité géographique, leur lenteur» (LORRAIN, 1977, p. 359). Leurs reproches se concentrent sur deux aspects de la gestion du personnel : le «recrutement social»d'une part, la «promotion mairie» de l'autre. Le recrutement social désigne la motivation assistantielle de certaines embauches, visant à secourir, par l'accès à un emploi souvent peu qualifié et peu rémunérateur, des personnes repérées comme en difficulté sociale ou familiale. Prenant des formes variées au cours du temps (du recrutement prioritaire des anciens combattants à celui des femmes divorcées) et selon la couleur politique des exécutifs locaux, il est devenu le symbole des travers du recrutement local - accusé de faire passer au second plan le critère de la compétence. Quant aux «promotions mairie», fussent-elles adossées sur des compétences acquises en formation continue, elles sont suspectées d'entretenir les idiosyncrasies locales, au détriment des outils de gestion, orientés vers «l'efficience», diffusés à partir des années 1980 (BILAND, 2009).

On ne saurait en conclure que les maires ont été rapidement convaincus par la pertinence de ces changements. La correspondance entre le territoire d'élection et le marché local du travail forge en effet

(6) En $1969,1,5 \%$ des agents communaux avaient un niveau supérieur au baccalauréat, contre $8,3 \%$ de la population active (LORRAIN, 1988, p. 88). Ce différentiel tient pour partie à la différence de structure hiérarchique (avec une surreprésentation des emplois d'exécution dans la FPT), mais jusque dans les années 1980, il est aussi observé entre des fonctionnaires ayant des fonctions similaires. l'intérêt réciproque des acteurs : les élus embauchent leurs électeurs et répondent à des demandes d'emploi croissantes avec l'augmentation du chômage; les habitants peuvent travailler sur place et y faire valoir, en particulier dans les couches populaires, leur capital d'autochtonie (RETIÈRE, 2003). De surcroît, les nouveaux embauchés ont régulièrement une expérience d'ouvriers qualifiés dans des entreprises locales, ce qui en fait une main-d'œuvre attractive pour les communes. Mais depuis le début des années 1980, trois facteurs ont rendu souhaitable la mobilité géographique parmi les élites locales : le rapprochement juridique avec la fonction publique de l'Etat; les transformations du paysage institutionnel local qui renouvelle la morphologie de la FPT; les pressions à la maîtrise des finances publiques enfin.

\section{Vers un «marché " territorial?}

Parler de marché pour qualifier la fonction publique territoriale peut doublement étonner. En premier lieu, ce terme s'oppose à la régulation statutaire supposée caractériser la fonction publique française, relevant du système de la carrière ${ }^{(7)}$. La FPT, on le sait, est toutefois bien loin de ce modèle. En particulier, les très nombreux employeurs ${ }^{(8)}$ y choisissent directement leurs agents, quand bien même ceux-ci seraient lauréats de concours. Deuxième contreindication à l'usage de la métaphore marchande : la faible mobilité des agents publics locaux contrevient au critère de fluidité. Cette réserve a partiellement été levée depuis le début des années 1980. Les évolutions juridiques, morphologiques et gestionnaires de la FPT ont encouragé les circulations entre employeurs, contribuant à la construction d'un marché interne ${ }^{(9)}$, non plus à l'échelle d'une collectivité mais à celle des employeurs publics locaux dans leur ensemble. Ce marché n'en demeure pas moins marqué d'incomplétude : de multiples segmentations - géographiques et politiques, tout particulièrement - en limitent l'unification. En somme, si plusieurs tendances se révèlent favorables à la mobilité entre collectivités, voire entre FPT et FPE et même entre public et privé, celles-ci restent limitées. Leur développement fait figure d'horizon politique et managérial, inscrit dans le vaste mouvement de réforme des administrations publiques dont la FPT est supposée être le «laboratoire» $(10)$.

(7) Dans ce système, l'agent est recruté, non pour occuper un emploi particulier, éventuellement temporaire, mais pour suivre une carrière, marquée des différentes formes d'avancement ainsi que par des changements d'activité. En effet, le grade est garanti, mais le poste, lui, peut changer (AuBy et al., 2005 , p. 11 et suiv.).

(8) Il y a aujourd'hui plus de 57000 employeurs publics locaux pour environ 2 millions d'agents (DGCL, 2011, p. 103). (9) Usuellement définie comme l'unité institutionnelle à l'intérieur de laquelle les salaires, les recrutements et les mobilités sont déterminés par des procédures administratives plutôt que marchandes (PIORE, Doringer, 1971).

(10) Brice Hortefeux, alors ministre délégué aux collectivités territoriales, à l'Assemblée Nationale, octobre 2006. 
Du côté des évolutions juridiques, c'est évidemment l'unification du statut des agents publics locaux et leur intégration, de plein droit, dans le giron de la fonction publique qu'il faut mentionner. En 19831984, la création de la fonction publique territoriale apparaît comme une contrepartie, sous la forme de protections statutaires accordées aux agents, aux prérogatives octroyées aux élus locaux par la décentralisation (SCHRAMECK, 1995, pp. 14-15; DuRAND, 2004, pp. 222-225). Les lois du 13 juillet 1983 et du 16 janvier 1984 favorisent la mobilité à l'intérieur de la fonction publique : les mutations sont désormais ouvertes entre tous les types de collectivités et d'établissements publics locaux; le détachement et la mise à disposition sont possibles vers une administration d'Etat ${ }^{(11)}$. De surcroît, plusieurs milliers de fonctionnaires des préfectures et des directions départementales de l'Équipement deviennent à cette occasion fonctionnaires des conseils généraux et régionaux - un mouvement renouvelé en 20062008 dans la foulée de $1^{\prime}$ « acte II» (12). Au-delà de ce cadre juridique, c'est bien la dynamique des institutions publiques locales qui s'est révélée favorable. Outre la croissance des institutions départementales et régionales, la montée en puissance des intercommunalités, depuis les années 1990, a concouru à la diminution de la part de l'emploi communal (qui ne représente plus que $56 \%$ du total). Par ailleurs, la loi de 1984 a créé plusieurs institutions (CNFPT et centres départementaux de gestion) qui rapprochent les pratiques de recrutement et de gestion et offrent un cadre de socialisation commun aux fonctionnaires territoriaux - rendant plus aisée, y compris subjectivement, la projection dans un autre emploi. Le renforcement des cadres d'emplois de catégorie A a d'ailleurs accru le rôle des associations professionnelles ${ }^{(13)}$, au sein desquelles se construisent les réseaux utiles à la mobilité entre employeurs.

Les changements juridiques et gestionnaires intervenus depuis la fin des années 1980 ont vraisemblablement diminué l'intérêt des élus pour le personnel pérenne. Moins contraints à recruter des fonctionnaires, encouragés à contrôler leur masse salariale dans une optique de maitrise des finances locales ${ }^{(14)}$, ils ont aussi plus de facilité pour attirer des «anciens du privé». Des concours (troisième voie) leur sont dédiés et leur ancienneté est maintenant partiellement prise en compte. Certes, le recrutement local persiste, en particulier en catégorie $\mathrm{C}$. Toutefois, il est de moins en moins social : exception faite des emplois aidés ${ }^{(15)}$, les embauches aux postes les moins qualifiés sont nettement moins fréquentes qu'auparavant (Le Lidec, Montricher, 2004). La tendance à la privatisation de la fonction publique, entendue comme le rapprochement du droit de la fonction publique à l'égard du code du travail et plus largement comme l'adoption, dans le public, de pratiques gestionnaires attribuées au secteur privé (Delvové, 2006), est finalement un facteur de mobilité externe. Toutefois, la création du «CDI public» (par la loi dite Dutreil du 26 juillet $2005^{(16)}$ ) semble peu conforme à cet objectif. En effet, elle permet à certains contractuels d'obtenir un emploi stable (via un contrat à durée indéterminée de droit public), sans toutefois disposer des possibilités de mutation, accessibles aux seuls fonctionnaires.

Par ailleurs, la politisation structurelle des cadres territoriaux subit des changements au regard de cette norme contemporaine de mobilité géographique. Alors que la loyauté due par le secrétaire général au maire était auparavant forgée au cours d'une relation de longue durée, celle-ci est progressivement devenue contractuelle et à durée déterminée. Le détachement sur des emplois fonctionnels permet en effet de lier le contrat du directeur général des services (DGS) et de ses adjoints aux échéances électorales. Signalé pour la première fois après les élections municipales de 1977 (marquées par la victoire de la gauche), le système des dépouilles territorial est devenu un marronnier pour la presse spécialisée, qui n'hésite pas à parler de mercato à son endroit (Le SaOut, 2011, p. 4). Si l'on observe effectivement une forte augmentation de la mobilité des DGS depuis le milieu des années 1990 (ibid, pp. 6-7), on sait aussi que les changements à la tête des exécutifs locaux n'en sont pas exclusivement à l'origine. Beaucoup de cadres territoriaux ont aujourd'hui intégré la mobilité entre collectivités comme une condition de leur ascension professionnelle. À peine plus d'un cadre supérieur sur dix a fait toute sa carrière au sein de la même collectivité

(14) Parmi les ratios définis par la loi du 6 février 1992 pour rationaliser et rendre plus transparente la gestion locale, le rapport entre les dépenses de personnel et les dépenses de fonctionnement figure d'ailleurs en bonne place (LE LIDEC, 2006, p. 39).

(15) Ces contrats de droit privé concernent 57000 agents en 2009 (DGCL, 2010, p. 1).

(16) Les dispositions de cette loi sont confirmées par le projet de loi relatif à l'accès à l'emploi titulaire et à l'amélioration des conditions d'emploi des agents contractuels dans la fonction publique présenté en conseil des ministres le 7 septembre 2011, lequel prévoit toutefois des possibilités d'accès au statut de fonctionnaire. 
(BAChelet, 2006). Permises par le statut, les mutations sont facilitées par la publicité nationale des offres d'emploi (dans la Gazette des communes, la Lettre du cadre territorial, Le Moniteur, Télérama), mais aussi par les relations d'interconnaissance qui lient cadres et élus des plus grandes collectivités, au nombre réduit (LE SAOUT, 2011, p. 11).

Cette mobilité géographique distingue les cadres des autres catégories (KouBI, 2005, p. 77). Les mutations représentent $25 \%$ des recrutements de cadres A intervenus en 2007, contre $7 \%$ des embauches en catégorie C (OBSERVATOIRE, 2010, p. 3). L'autonomisation à l'égard des canaux locaux de l'embauche singularise le recrutement des cadres de celui des agents d'exécution, pour lequel les collectivités utilisent souvent les services de Pôle Emploi. Elle différencie les personnes issues des classes populaires, dont les voies historiques de promotion se sont construites au local, et les personnes issues des classes moyennes, tôt confrontées, par leurs études et par leurs alliances matrimoniales ${ }^{(17)}$, à la mobilité spatiale. Avec $78 \%$ d'agents en catégorie C (DGCL, 2011, p. 105), la structure de la FPT surreprésente les emplois d'exécution. Combinée à la préférence historique pour le «local», cette morphologie explique sans doute que les agents territoriaux demeurent peu mobiles géographiquement, comparés à ceux des autres secteurs. Au cours des années 1990, ils ont été $2 \%$ en moyenne chaque année à changer de territoire de travail, contre $4 \%$ à l'État et $13 \%$ dans le privé (KouBI, 2005, p. 69; Pauron, 2003, p. 93). Ainsi que nous allons à présent le montrer, la norme contemporaine de mobilité pèse de manière inégale sur les groupes sociaux selon les coûts, économique, symbolique, relationnel, auxquels elle est associée.

\section{L'inscription sociale, genrée et générationnelle des mobilités passées des agents d'une petite ville de province}

Pour affiner ces tendances générales, et étudier les facteurs précis qui orientent les parcours professionnels, l'échelle monographique se révèle appropriée. Sous réserve de situer la collectivité étudiée au regard des indicateurs (strate démographique, couleur politique, bassin d'emploi, place dans l'armature urbaine) qui la caractérise, elle permet d'étudier en finesse des dynamiques trop souvent agglomérées par les données nationales. En analysant, au moyen de régressions logistiques rétrospectives, les différents types de mobilité passées (entre

(17) Rappelons que les cadres constituent le groupe social au sein duquel la rencontre du conjoint sur le lieu des études est le plus fréquent (Bozon, Héran, 2006, pp. 64-67). services, catégories hiérarchiques et employeurs) dont les agents d'une petite ville provinciale ont fait l'expérience ( $c f$. encadré 1), on se donne l'occasion d'examiner la structure générationnelle, genrée et sociale de leurs carrières ( $c f$. tableau 1).

\section{Encadré 1 \\ Présentation de la collectivité étudiée et des données recueillies}

Cinquième ville de son département, Tozay est une cité historiquement industrielle. Toutefois, depuis le début des années 1970, plusieurs restructurations ont conduit à la diminution de sa population (de 20000 habitants il y a quarante ans à 15000 aujourd'hui). La tertiarisation du marché local du travail se traduit notamment par la montée en puissance de l'emploi public : les collectivités (mairie) et établissements publics (hôpital) figurent désormais en tête des employeurs. Ici comme ailleurs (LE LIDEC, MontRicher, 2004, p. 22), la position de ville-centre, touristique de surcroît, renforce également les effectifs communaux, qui comptent $\mathbf{3 7 6}$ fonctionnaires et contractuels affectés sur des emplois permanents au moment de l'enquête (2006-2007).

Cette dernière repose sur des entretiens avec des élus et des agents et sur l'exploitation des dossiers de carrière de tous ces agents, conservés au service du personnel. Les arrêtés qu'ils contiennent permettent de retracer la carrière des agents, tandis que les données d'état civil et les diplômes fournissent des indicateurs sociodémographiques. Les agents les plus anciens de la commune ont été recrutés au début des années 1970, au moment où l'«union de la gauche» a accédé aux responsabilités, qu'elle a conservées jusqu'en 1995. Dirigée par un communiste, cette majorité a alors été battue par la droite, avant une nouvelle victoire de la gauche aux élections de 2001. En d'autres termes, la grande majorité $(78 \%)$ de ces agents a été recrutée alors que l'exécutif municipal était marqué à gauche.

La base de données constituée à partir de ces dossiers regroupe plusieurs cohortes de recrutement, dont il convient toutefois de noter le caractère incomplet puisque les personnes qui ont quitté la ville après y avoir travaillé quelques années n'apparaissent pas dans la base. Son appréhension de la mobilité géographique est donc partielle : sont définis comme mobiles géographiquement les individus qui ont travaillé pour un autre employeur public avant d'être embauché à Tozay. En d'autres termes, cette enquête ne renseigne pas les mobilités au départ de Tozay, vers le privé ou vers d'autres employeurs publics. En revanche, elle permet d'éclairer la composition actuelle d'une administration communale de quelques centaines d'agents.

Les caractéristiques de ces 376 agents sont présentées dans le tableau 1.

(1) Deux agents ont travaillé pour un autre employeur public (l'hôpital) de Tozay avant de travailler à la mairie. Toutefois, on les considère comme suffisamment peu nombreux pour considérer cette expérience d'un autre employeur public comme un proxy de la mobilité spatiale dans la fonction publique. 
Tableau 1 : Qui sont les agents de la ville de Tozay?

\begin{tabular}{|c|c|}
\hline Caractéristiques des agents & Part dans l'ensemble \\
\hline \multicolumn{2}{|l|}{ Lieu de naissance } \\
\hline Tozay & $56 \%$ \\
\hline Département hors Tozay & $16 \%$ \\
\hline Autre département & $28 \%$ \\
\hline \multicolumn{2}{|l|}{ Sexe } \\
\hline Hommes & $61 \%$ \\
\hline Femmes & $39 \%$ \\
\hline \multicolumn{2}{|l|}{ Âge } \\
\hline Moins de 30 ans & $7 \%$ \\
\hline 30 à 39 ans & $20 \%$ \\
\hline 40 à 49 ans & $40 \%$ \\
\hline 50 ans et plus & $33 \%$ \\
\hline \multicolumn{2}{|l|}{ Diplôme } \\
\hline Pas de diplôme, CEP ou BEPC & $24 \%$ \\
\hline BEP ou CAP & $45 \%$ \\
\hline Baccalauréat et plus & $31 \%$ \\
\hline \multicolumn{2}{|l|}{ Concours } \\
\hline Jamais passé & $47 \%$ \\
\hline Déjà passé & $53 \%$ \\
\hline \multicolumn{2}{|l|}{ Âge à l'entrée dans l'emploi public } \\
\hline Moins de 30 ans & $58 \%$ \\
\hline 30 à 39 ans & $30 \%$ \\
\hline 40 à 49 ans & $10 \%$ \\
\hline 50 ans et plus & $2 \%$ \\
\hline \multicolumn{2}{|l|}{ Catégorie hiérarchique en 2006} \\
\hline $\mathrm{C}$ & $80 \%$ \\
\hline B & $14 \%$ \\
\hline À & $6 \%$ \\
\hline \multicolumn{2}{|l|}{ Filière en 2006} \\
\hline Technique & $59 \%$ \\
\hline Administrative & $21 \%$ \\
\hline Autre filière & $20 \%$ \\
\hline
\end{tabular}

Note de lecture : $56 \%$ des agents municipaux de Tozay y sont nés, contre $16 \%$ dans le département et hors de Tozay, et $28 \%$ dans un autre département.

Champ : agents titulaires, stagiaires et contractuels permanents de la Ville de Tozay en 2006-2007.

Source : base de données constituée à partir des dossiers du personnel en 2006-2007 ( $\mathrm{n}=376)$.

\section{Fonctionnelle, hiérarchique, spatiale : trois formes de mobilité}

Près d'un tiers des agents tozayiens (29\%) a déjà changé de service, ce qui en fait l'expérience de mobilité la plus fréquente dans cette commune. Sans surprise, cette mobilité horizontale ${ }^{(18)}$ est d'autant plus probable que l'on a été embauché jeune, comme l'indique le tableau 2. Mais, quand on sait que les agents ont été embauchés depuis un peu plus de quatorze ans en moyenne, on ne peut que relativiser la mobilité entre services. Malgré le temps passé à travailler pour la mairie, les occasions d'en changer restent relativement peu fréquentes. Qui sont donc ces agents mobiles en interne? A priori associé aux mobilités verticales, le passage du concours se révèle également favorable à la mobilité

(18) N'étant pas directement liés à la logique administrative qui préside à la constitution des dossiers de carrière, les changements de postes et de fonctions ne sont que très imparfaitement indiqués dans les dossiers. C'est pourquoi j'ai retenu la mobilité entre services, mieux renseignée, pour estimer la mobilité horizontale au sein de la collectivité. horizontale. $67 \%$ des personnes «mobiles» d'un service à l'autre ont passé un concours, contre $27 \%$ des personnes ayant toujours travaillé dans le même service. Or, même s'il n'est pas toujours associé à une mobilité statutaire, le changement de service se traduit souvent par une évolution des tâches, qui requiert de nouvelles compétences. Au cours des entretiens menés avec des agents, la plupart de ces changements étaient présentés comme volontaires et associés à un intérêt accru au travail. Le changement de service renvoie également aux petites mobilités sociales qui ont valu à la «territoriale» sa réputation de fonction publique promotionnelle (BILAND, 2010b, pp. 135-136). Ni le diplôme, ni la catégorie ne sont d'ailleurs des caractéristiques significativement associées à ces formes passées de mobilité, signe possible des petits déplacements auxquels elles sont associées. Signe également que les agents que nous rencontrons à Tozay ont pu y rester parce qu'ils sont parvenus à y obtenir une mobilité horizontale, ou que ceux qui en sont partis ont obtenu de plus amples promotions. En tout cas, parce que les postes accessibles aux personnes peu gradées sont structurellement plus nombreux, parce que les travaux les plus pénibles peinent à être exercés des décennies durant, la mobilité entre services apparaît socialement moins inégalitaire que les autres.

La nature des fonctions rend tout de même plus ou moins probable cette mobilité. Ce sont les agents de la filière administrative qui ont le plus de chance d'avoir changé de service. Leur polyvalence (sensible dans la variété des tâches assumées par une secrétaire, par exemple) faciliterait le passage d'un service à un autre ${ }^{(19)}$. La féminisation des emplois administratifs (70 \% à Tozay) ne doit toutefois pas laisser croire que les femmes ont davantage accès à cette mobilité fonctionnelle. Le Tableau 2 suggère le contraire puisque les femmes en poste en 2006 ont moins souvent connu de changements de service en cours de carrière que leurs homologues masculins. Cette observation statistique concorde avec les récits recueillis en entretien. Au sein de plusieurs couples de "communaux», la réussite professionnelle de l'homme s'est appuyée sur le soutien de son épouse, elle-même engagée au travail sur un mode subordonné aux charges familiales.

Les dossiers de carrière indiquent également l'existence d'une corrélation négative entre mobilité interne et mobilité spatiale. On a d'autant plus

(19) Soulignons, à l'appui de cet argument, que seulement 16 agents (soit $4 \%$ des effectifs) ont connu un changement de filière depuis leur arrivée à Tozay. Il s'agit la plupart du temps d'un départ de la filière technique pour la filière administrative $(5 \mathrm{cas})$ et surtout la filière sociale $(9$ cas $)$. Deux changements de filière renvoient à des évolutions marquées du travail (du ménage dans les écoles au service des finances, par exemple), mais il s'agit alors d'une régularisation liée à la création d'un nouveau cadre d'emplois (celui des ATSEM) ou d'une petite mobilité fonctionnelle à l'intérieur d'un même milieu de travail (de femme de ménage dans les écoles à ATSEM). 
de chance d'avoir connu un changement de service que l'on est né sur place : l'appartenance locale semble bien avoir favorisé, pour les agents restés à Tozay, l'inscription dans un parcours «mairie». De la même manière, le fait d'avoir connu un autre employeur public est associé négativement aux changements de service, ce qui renvoie à une forme d'exclusive entre carrières internes et externes.

\section{Deux trajectoires "couplées" dans la filière administrative}

Les carrières respectives de Gilles et Maryse Moine illustrent ces inégalités genrées au sein de la filière administrative. Fils de cordonnier, bachelier, Gilles entre à la mairie en 1972, après un an passé à la préfecture. II est alors commis (catégorie C) et devient rédacteur (B) en 1975, puis attaché (A), en 1979, les deux fois par concours. Il dirige ensuite, jusqu'à sa retraite en 2004, plusieurs services et atteint le grade d'attaché principal par promotion interne. Après avoir travaillé en supermarché et donné naissance à leur premier enfant, sa conjointe l'y rejoint en 1977. Moins diplômée que lui, il est vrai (elle a un BEPC), elle travaille depuis lors au service de l'état civil et des élections. Recrutée comme téléphoniste (bas de la catégorie $\mathrm{C}$ ), elle ne passe pas de concours et travaille longtemps à temps partiel. Promue à l'ancienneté adjointe administrative (nouvelle dénomination des commis) en 1990, elle ne parvient pas à accéder au cadre d'emplois de rédacteur (catégorie $B$ ).

Examinant à présent les facteurs corrélés avec le changement passé de catégorie hiérarchique (de $\mathrm{C}$ à $\mathrm{B}$ ou de $\mathrm{B}$ à $\mathrm{A}$, voire de $\mathrm{C}$ à $\mathrm{A}$ ), on s'intéresse à une forme de mobilité explicitement verticale et beaucoup moins fréquente ( $7 \%$ des agents). Comme l'indique le tableau 3, il y a «toutes choses égales par ailleurs» plus de bacheliers parmi les agents qui ont «grimpé». Ce constat témoigne du rôle du diplôme dans la sélection interne des agents, par exemple pour des promotions venant compenser un déclassement en début de vie active(20). Même dans un milieu réputé promotionnel, les prérequis scolaires à la promotion sont significatifs ${ }^{(21)}$. On remarque ainsi que les cinq agents ayant commencé leur carrière en catégorie $\mathrm{C}$ et ayant atteint la catégorie $\mathrm{A}$ au moment de l'enquête sont tous bacheliers : quatre sont même

(20) Étonnante, la non significativité du facteur «concours» peut s'expliquer par cette prégnance du diplôme : à diplôme donné, le passage du concours n'augmente pas significativement les chances de changer de catégorie hiérarchique, même si l'effet «brut» du concours sur ce type de mobilité est évidemment positif : 21 des 27 personnes ayant changé de catégorie hiérarchique en cours de carrière - soit $78 \%$ - ont déjà passé des concours. Par contraste, seules $49 \%$ des personnes n'ayant pas changé de catégorie hiérarchique ont l'expérience du concours. (21) De manière générale, on sait que les titulaires de diplômes généraux sont plus nombreux à passer de la position ouvriers / employés aux positions professions intermédiaires / cadres (Chapoulie, 2000, pp. 42-43). diplômés du supérieur alors qu'ils sont relativement âgés (quatre quinquagénaires sur cinq).

Tableau 2 : Quels agents ont déjà changé de service depuis l'embauche en mairie?

\begin{tabular}{|c|c|c|}
\hline $\begin{array}{c}\text { Caractéristiques } \\
\text { des agents }\end{array}$ & $\mid \begin{array}{c}\text { Part de } \\
\ll \text { mobiles» }\end{array}$ & $\begin{array}{l}\text { Odds } \\
\text { ratio }\end{array}$ \\
\hline \multicolumn{3}{|l|}{ Lieu de naissance } \\
\hline Tozay & $37 \%$ & Réf \\
\hline Département hors Tozay & $17 \%$ & 0,35 \\
\hline Autre département & $15 \%$ & \\
\hline \multicolumn{3}{|l|}{\begin{tabular}{|l|} 
Sexe \\
\end{tabular}} \\
\hline Hommes & $39 \%$ & Réf \\
\hline Femmes & $28 \%$ & 0,4 \\
\hline \multicolumn{3}{|l|}{ Âge } \\
\hline Moins de 30 ans & $4 \%$ & \\
\hline 30 à 39 ans & $27 \%$ & \\
\hline 40 à 49 ans & $28 \%$ & \\
\hline 50 ans et plus & $34 \%$ & \\
\hline \multicolumn{3}{|l|}{\begin{tabular}{|l|} 
Diplôme \\
\end{tabular}} \\
\hline Pas de diplôme, CEP ou BEPC & $33 \%$ & Réf \\
\hline BEP ou CAP & $40 \%$ & \\
\hline Baccalauréat et plus & $29 \%$ & \\
\hline \multicolumn{3}{|l|}{\begin{tabular}{|l|} 
Autre employeur public \\
\end{tabular}} \\
\hline Jamais & $30 \%$ & Réf \\
\hline Avant Tozay & $12 \%$ & 0,41 \\
\hline \multicolumn{3}{|l|}{ Concours } \\
\hline Jamais passé & $27 \%$ & Réf \\
\hline Déjà passé & $41 \%$ & 2,0 \\
\hline \multicolumn{3}{|c|}{ Âge à l'entrée dans l'emploi public } \\
\hline Moins de 30 ans & $35 \%$ & $0,97^{*}$ \\
\hline 30 à 39 ans & $31 \%$ & \\
\hline 40 à 49 ans & $32 \%$ & \\
\hline 50 ans et plus & $20 \%$ & \\
\hline \multicolumn{3}{|l|}{ Catégorie hiérarchique en 2006} \\
\hline $\mathrm{C}$ & $31 \%$ & \\
\hline $\mathrm{B}$ & $17 \%$ & \\
\hline$\grave{A}$ & $20 \%$ & \\
\hline \multicolumn{3}{|l|}{ Filière en 2006} \\
\hline Technique & $29 \%$ & Réf \\
\hline Administrative & $32 \%$ & 2,4 \\
\hline Autre filière & $18 \%$ & \\
\hline Ensemble & $29 \%$ & \\
\hline
\end{tabular}

* Pour ce tableau, comme pour les deux suivants, les odd-ratio sur l'âge en 2006 et l'âge à l'entrée dans l'emploi public sont calculés sur des variables continues. Dans ce cas, l'effet de l'âge à l'entrée dans l'emploi public sur la mobilité entre services se lit de la manière suivante plus on est entré tard dans l'emploi public, moins on a de chances d'avoir connu une mobilité entre les services de la mairie de Tozay.

Note de lecture : $39 \%$ des hommes ont changé de service au cours de leur carrière d'agent de Tozay, contre $28 \%$ des femmes, qui ont ainsi, «toute autre caractéristique du modèle étant prise en compte par ailleurs», 0,41 fois moins de chance relative d'avoir connu une telle mobilité que leurs homologues masculins.

Champ : agents titulaires, stagiaires et contractuels permanents de la Ville de Tozay en 2006-2007.

Source : base de données constituée à partir des dossiers du personnel en 2006-2007 ( $\mathrm{n}=376)$.

Les deux variables liées à l'âge semblent plus heuristiques. Les personnes plus âgées, et entrées plus jeunes en collectivité, ont plus de chance d'avoir connu une ascension professionnelle. De fait, les mobilités de forte amplitude prennent du 
temps, comme le montrent les différentes étapes franchies par Gilles Moine pour passer du bas au sommet de l'échelle. S'il faut avoir passé du temps en collectivité, c'est peut-être aussi parce que les conditions actuelles de gestion des carrières autorisent moins qu'avant la mobilité verticale au sein de la même collectivité. La comparaison des parcours des quinquagénaires et quadragénaires est à cet égard frappante. Les plus jeunes sont deux fois moins nombreux que leurs aînés à avoir changé de catégorie hiérarchique (7\% contre $13 \%)$. Connaîtront-ils les mêmes succès dans les prochaines années? Rien n'est moins sûr, car les changements de catégorie se font la plupart du temps autour de 40 ans. En fait, le destin différent de ces deux cohortes, éloignées de quelques années seulement, semble correspondre à une césure intervenue au milieu des années 1990 : dans les collectivités de la taille de Tozay, le transfert des concours et des promotions à des institutions départementales, les centres de gestion, augmente la sélectivité de ces deux dispositifs (BILAND, 2009, p. 25). Le rapprochement, récent mais rapide, des niveaux de diplôme entre la FPT et la FPE (22), contribue d'ailleurs à renchérir les prérequis scolaires à l'ascension professionnelle.

L'appartenance locale est également un facteur significatif pour ce type de mobilité, mais en sens inverse de la mobilité entre services déjà étudiée. Ainsi, ce sont les personnes nées hors du département où se trouve Tozay, qui ont, à autres caractéristiques données, le plus de chances d'avoir changé de catégorie hiérarchique.

Que penser de la spécificité de la filière administrative? Les «administratifs» cumuleraient-ils mobilités verticale et horizontale? Les carrières des individus qui ont connu les parcours les plus promotionnels (à l'instar de Gilles Moine) semblent l'indiquer : leurs changements de service suivent en général de peu leur réussite au concours et leur accès à un cadre d'emplois plus élevé. En d'autres termes, les grandes mobilités (au sens fonctionnel et hiérarchique) sont caractéristiques de la filière administrative, mais elles demeurent rares. Par contraste, les agents de la filière technique ont accès, en grand nombre, à des mobilités hiérarchiques plus modestes (changement de grade à l'intérieur d'une catégorie donnée).
Tableau 3 : Qui sont les agents ayant changé de catégorie en cours de carrière?

\begin{tabular}{|c|c|c|}
\hline Caractéristiques des agents & $\begin{array}{c}\text { Part de } \\
\text { «mobiles } »\end{array}$ & $\begin{array}{l}\text { Odds } \\
\text { ratio }\end{array}$ \\
\hline \multicolumn{3}{|l|}{ Lieu de naissance } \\
\hline Tozay & $5 \%$ & Réf \\
\hline Département hors Tozay & $5 \%$ & \\
\hline Autre département & $22 \%$ & 2,5 \\
\hline \multicolumn{3}{|l|}{ Sexe } \\
\hline Hommes & $8 \%$ & \\
\hline Femmes & $6 \%$ & \\
\hline \multicolumn{3}{|l|}{ Âge } \\
\hline Moins de 30 ans & $0 \%$ & 4,2 \\
\hline 30 à 39 ans & $3 \%$ & \\
\hline 40 à 49 ans & $7 \%$ & \\
\hline 50 ans et plus & $13 \%$ & \\
\hline \multicolumn{3}{|l|}{ Diplôme } \\
\hline Pas de diplôme, CEP ou BEPC & $5 \%$ & Réf \\
\hline BEP ou CAP & $2 \%$ & \\
\hline Baccalauréat et plus & $17 \%$ & 4,3 \\
\hline \multicolumn{3}{|l|}{ Autre employeur public } \\
\hline Jamais & $5 \%$ & \\
\hline Avant Tozay & $28 \%$ & \\
\hline \multicolumn{3}{|l|}{ Concours } \\
\hline Jamais passé & $4 \%$ & \\
\hline Déjà passé & $11 \%$ & \\
\hline \multicolumn{3}{|l|}{ Âge à l'entrée dans l'emploi public } \\
\hline Moins de 30 ans & $9 \%$ & 0,89 \\
\hline 30 à 39 ans & $5 \%$ & \\
\hline 40 à 49 ans & $0 \%$ & \\
\hline 50 ans et plus & $17 \%$ & \\
\hline \multicolumn{3}{|l|}{ Filière en 2006} \\
\hline Technique & $5 \%$ & Réf \\
\hline Administrative & $14 \%$ & 4,0 \\
\hline Autre filière & $8 \%$ & \\
\hline Ensemble & $7 \%$ & \\
\hline
\end{tabular}

Note de lecture : $5 \%$ des agents de la filière technique, contre $14 \%$ des agents de la filière administrative ont changé de catégorie hiérarchique. Ces derniers ont ainsi, «toute autre caractéristique du modèle étant prise en compte par ailleurs», 4,0 fois plus de chance relative d'avoir connu une telle mobilité que leurs homologues de la filière technique.

Champ : agents titulaires, stagiaires et contractuels permanents de la Ville de Tozay en 2006-2007.

Source : base de données constituée à partir des dossiers du personnel en 2006-2007 $(\mathrm{n}=376)$.

$\mathrm{Au}$ terme de l'étude de ces deux premières formes de mobilité, on observe finalement une polarisation opposant, d'un côté, des agents venus d'ailleurs, davantage diplômés ${ }^{(23)}$, qui ont plus souvent connu une mobilité hiérarchique et travaillent dans la filière administrative, de l'autre des agents « du cru», appartenant plus fréquemment à la filière
(22) Notamment sensibles dans les profils des candidats aux concours, régulièrement surdiplômés par rapport aux exigences officielles.
(23) $56 \%$ des agents nés hors du département sont bacheliers, contre $20 \%$ des agents nés à Tozay et $27 \%$ de ceux nés dans ce département. 
technique et dont la mobilité est interne(24). Nous affinons l'analyse en examinant les déterminants de la mobilité entre collectivités.

L'articulation entre position territoriale et position sociale s'enrichit avec l'analyse de la mobilité entre employeurs publics ( $c f$. tableau 4). Les agents tozayiens ne font pas exception au mode d'entrée dans l'emploi public local rappelé dans la première partie : $60 \%$ des enquêtés ont travaillé dans le secteur privé ( $10 \%$ en CDI, $50 \%$ en CDD ou intérim) avant de devenir fonctionnaire. L'âge moyen d'entrée en collectivité est proche de 30 ans. Ces passages privépublic se doublent-ils de mobilités entre collectivités? Pour revenir sur l'hypothèse du marché interne aux emplois publics locaux, une dernière analyse compare les individus ayant travaillé pour d'autres employeurs publics avant de venir à Tozay à ceux pour qui cette mairie est le premier employeur public. L'ampleur de ce dernier type de mobilité est limitée : $12 \%$ des agents ont déjà travaillé pour au moins une autre collectivité, et $2 \%$ pour l'État. Il est vrai que les données recueillies ne donnent accès qu'à une partie des changements d'employeur : elles ne permettent pas de suivre les agents tozayiens qui sont partis travailler ailleurs. Tout de même, seulement $6 \%$ des personnes recrutées avant 1995 ont préalablement travaillé pour un autre employeur public. Jusque dans les années 1990, les mobilités entre emploi public et emploi privé, le plus souvent au sein de la même scène locale, sont plus fréquentes que les mobilités entre employeurs publics, synonymes de mobilité spatiale. Les bilans sociaux comptabilisent vingt-cinq démissions intervenues entre 1986 et 1993 , contre seulement quatre mutations au cours de la même période. Les choses ont visiblement changé depuis : $18 \%$ des agents recrutés depuis 1995 avaient déjà une expérience dans le public, et ce sont même $27 \%$ des personnes arrivées depuis 2001 qui sont dans ce cas. À l'échelle nationale, au cours des années 1990, ce sont 2 à $3 \%$ des agents qui changent de collectivité une année donnée (KouBi, 2005, p. 73). Sans surprise, les mutations sont d'autant moins fréquentes que la taille de l'administration est importante : avec près de 400 agents, l'administration tozayienne a déjà atteint un développement suffisant pour offrir des perspectives de carrière en interne. À cet égard, la filière administrative, où les emplois sont moins nombreux et moins spécifiques que dans la filière technique, fait exception : elle est particulièrement propice à la mobilité entre collectivités ( $c f$. tableau 4).

(24) La non-significativité de la variable «sexe» s'agissant de la mobilité hiérarchique laisse penser qu'il n'y a pas de différence entre hommes et femmes dans l'accès à la mobilité hiérarchique. Cette hypothèse contrevient aux observations de la sur-représentation des hommes dans l'encadrement, y compris au sein de l'encadrement promu (quatre des cinq agents passés de la catégorie $\mathrm{C}$ à la catégorie $\mathrm{A}$ sont par exemple des hommes). D'autres facteurs associés, comme la filière, qui agiraient différemment pour les hommes et les femmes, pourraient expliquer cette non significativité.
Tendanciellement croissante et inégalement fréquente selon les filières, cette forme de mobilité est surtout surdéterminée par le lieu de naissance des agents. Les personnes natives de Tozay ont fort peu de chances d'avoir travaillé pour un autre employeur public. A contrario, être né ailleurs et avoir passé un concours sont deux caractéristiques plus souvent partagées ${ }^{(25)}$ parmi ceux ayant travaillé pour un autre employeur public. La réalisation d'entretiens avec des candidats aux concours (BILAND, 2010a, p. 61) montre que ces derniers anticipent fortement le changement de collectivité, qui pourrait découler de leur réussite. Certains avouent d'ailleurs s'être découragés, s'étant progressivement rendu compte qu'il ne leur serait pas possible de «grimper sur place». Si le développement des concours, désormais organisés en dehors des collectivités, apparaît un facteur essentiel de montée en puissance des mutations, il peut aussi être tenu pour responsable de la raréfaction des trajectoires ascendantes au sein de classes populaires fortement attachées aux sociabilités locales (SchwARTz, 1998, p. 80).

La non-significativité du diplôme et de la catégorie hiérarchique semble pouvoir s'expliquer par la prégnance de l'effet «lieu de naissance» : parce que seulement $22 \%$ des catégories A sont nées à Tozay (contre $66 \%$ des C), parce que seulement $36 \%$ des bacheliers sont nés à Tozay (contre $66 \%$ des titulaires d'un CAP ou BEP), on peut considérer que le lieu de naissance s'impose sur ces deux caractéristiques qui lui sont largement corrélées.

Que penser, pour terminer, de la non-significativité des variables d'âge et d'ancienneté? On sait que la propension à la mobilité géographique est largement une affaire de cycle de vie : elle est, dans la FPT, dans la FPE comme dans le privé, particulièrement forte avant 30 ans, au cours de la période d'insertion sur le marché du travail (DuPAYS, 2003, p. 41 ; Pauron, 2003, p. 105 ; Koubi, 2005). Dans ces catégories d'âge, les mutations se sont banalisées, parce qu'elles fonctionnent comme une injonction en vue d'une carrière réussie, mais aussi parce qu'elles correspondent aux aspirations personnelles de nombreux jeunes actifs, encore dans une phase de transition entre leur famille d'orientation et leur famille de procréation, et qui ont, dès leurs études supérieures, connu la mobilité. Beaucoup en font une condition pour un intérêt maintenu au travail. En somme, pesant surtout sur les personnes en début et en milieu de vie active, l'injonction à la mobilité tend à réduire le poids de l'ancienneté. Les jeunes sont plus mobiles mais les plus âgés ont eu plus de temps pour changer d'employeur - ces deux effets croisés rendraient l'effet de ces variables non significatif. De surcroît, parmi les cohortes les plus

(25) Une analyse non publiée ici indique que la probabilité d'avoir passé un concours est plus forte chez les agents nés hors de Tozay. 
anciennement recrutées, un certain nombre d'agents sont partis travailler ailleurs. Or, l'exploitation des dossiers du personnel reste limitée à une seule collectivité - ces dossiers suivant les agents lorsque ceux-ci mutent. Finalement, si les plus âgés sont les moins mobiles, c'est aussi parce que les plus mobiles de leur génération ne travaillent plus à Tozay. Quant à la non-significativité de la variable «sexe», elle semble renvoyer aux motifs différents des hommes et des femmes amenés à changer de lieu de travail. Dans les lettres de motivation (conservées dans les dossiers) et les entretiens, plusieurs femmes indiquent souhaiter un rapprochement vis-à-vis de leur conjoint, tandis que les hommes semblent poursuivre un projet professionnel plus autonome.

Tableau 4 : Qui sont les agents ayant travaillé pour un autre employeur public?

\begin{tabular}{|c|c|c|}
\hline $\begin{array}{c}\text { Caractéristiques } \\
\text { des agents }\end{array}$ & $\begin{array}{c}\text { Part de } \\
\text { «mobiles» }\end{array}$ & $\begin{array}{l}\text { Odds } \\
\text { ratio }\end{array}$ \\
\hline \multicolumn{3}{|l|}{ Lieu de naissance } \\
\hline Tozay & $4 \%$ & Réf \\
\hline Département hors Tozay & $14 \%$ & 4,5 \\
\hline Autre département & $35 \%$ & 4,2 \\
\hline \multicolumn{3}{|l|}{ Sexe } \\
\hline Hommes & $11 \%$ & \\
\hline Femmes & $14 \%$ & \\
\hline \multicolumn{3}{|l|}{ Âge } \\
\hline Moins de 30 ans & $11 \%$ & \\
\hline 30 à 39 ans & $9 \%$ & \\
\hline 40 à 49 ans & $15 \%$ & \\
\hline 50 ans et plus & $10 \%$ & \\
\hline \multicolumn{3}{|l|}{ Diplôme } \\
\hline Pas de diplôme, CEP ou BEPC & $7 \%$ & Réf \\
\hline BEP ou CAP & $3 \%$ & \\
\hline Baccalauréat et plus & $30 \%$ & 4,3 \\
\hline \multicolumn{3}{|l|}{ Concours } \\
\hline Jamais passé & $8 \%$ & Réf \\
\hline Déjà passé & $19 \%$ & 2,6 \\
\hline \multicolumn{3}{|c|}{ Âge à l'entrée dans l'emploi public } \\
\hline Moins de 30 ans & 14 & \\
\hline 30 à 39 ans & 9 & \\
\hline 40 à 49 ans & 11 & \\
\hline 50 ans et plus & 17 & \\
\hline \multicolumn{3}{|l|}{ Catégorie hiérarchique en 2006} \\
\hline $\mathrm{C}$ & 5 & \\
\hline B & 30 & \\
\hline À & 52 & \\
\hline \multicolumn{3}{|l|}{ Filière en 2006} \\
\hline Technique & 5 & Réf \\
\hline Administrative & 19 & 2,9 \\
\hline Autre filière & 23 & \\
\hline Ensemble & $12 \%$ & \\
\hline
\end{tabular}

Note de lecture : $5 \%$ des agents de la filière technique ont déjà travaillé pour un autre employeur public, contre $19 \%$ des agents de la filière administrative, qui ont ainsi, «toute autre caractéristique du modèle étant prise en compte par ailleurs », 2,9 fois moins de chance relative d'avoir connu une telle mobilité que leurs homologues de la filière technique.

Champ : agents titulaires, stagiaires et contractuels permanents de la Ville de Tozay en 2006-2007.

Source : base de données constituée à partir des dossiers du personnel en 2006-2007 $(\mathrm{n}=376)$.

\section{Des ouvriers aux cadres : quatre trajectoires typiques}

Si les régressions ne suffisent à rendre compte de l'ensemble des motifs impliqués dans les mobilités, elles montrent de manière convaincante le poids de l'appartenance générationnelle, de la position sociale et du sexe sur les parcours professionnels dans la «territoriale». Au terme de cette analyse, on peut identifier quatre trajectoires modales à la mairie de Tozay, qui articulent genre, génération, position hiérarchique et spécialité professionnelle.

La première est typique des hommes, ouvriers qualifiés, titulaires de BEP/CAP, originaires de Tozay et qui connaissent localement de petites mobilités statutaires au sein d'un même atelier.

\section{Emploi technique et mobilité interne}

L'actuel responsable de la CGT, né en 1951, a connu cette ascension au sein de la catégorie C. Titulaire d'un BEP, il travaille une dizaine d'années comme ouvrier qualifié dans l'imprimerie, puis entre à la mairie en 1982, suite à un licenciement économique. Alors au grade le plus bas (aide ouvrier professionnel), il réussit quelques années plus tard le concours interne, organisé localement, d'agent technique peintre. Il est ensuite promu, à l'ancienneté, agent technique qualifié puis agent technique principal. En 2000, des problèmes de santé le conduisent à être reclassé dans un emploi administratif, au sein du même service.

Depuis le milieu des années 1990, ces parcours ascendants sont toutefois moins probables, en même temps qu'augmentent les exigences scolaires pour le recrutement initial (baccalauréat professionnel voire BTS).

Témoignant de la division genrée du travail, la deuxième trajectoire est le pendant féminin de la première. Elle est associée aux emplois administratifs et aux diplômes généraux intermédiaires (BEPC puis baccalauréat), marquée par une certaine mobilité entre services (sur des fonctions proches) et par une progression statutaire moindre.

\section{Emploi administratif et moindre progression statuaire}

Le parcours de l'actuelle secrétaire des services techniques en donne un bon exemple. En 1982, à l'issue de son école de secrétariat, cette dernière réussit le concours de commis et se trouve rapidement embauchée à la mairie de la ville de banlieue parisienne où elle est née. Elle y travaille comme animatrice pendant six ans, puis devient secrétaire du directeur des services techniques, poste qu'elle occupe pendant deux ans, avant d'obtenir une mutation pour Tozay. Motivée par une mutation antérieure de son époux, ce changement de région la conduit à occuper un poste similaire qu'elle ne quittera plus. 
La troisième trajectoire distingue les cadres des deux catégories d'agents d'exécution déjà évoquées. Contrairement à ces derniers, les «catégorie $\mathrm{A}$ » ont souvent fait l'expérience des concours dès le début de leur carrière, pour accéder au statut de fonctionnaire. Au-delà de cette expérience commune, leurs parcours se distinguent fortement selon le sexe, l'âge et le rapport au territoire. Au modèle de promotion interne, plutôt masculin, qui correspond aux agents les plus âgés, s'est substitué, depuis le milieu des années 1990, le recrutement des cadres par mutation. En 2006, 15 des 21 actuels «cadres A» de Tozay (soit $71 \%$ ) ont été recrutés au cours de la décennie précédant l'enquête. C'est dire si ce groupe a connu d'importantes transformations : plus jeunes, plus souvent femmes, ces cadres ont aussi des relations moins personnelles avec les élus.

\section{Promotion et mobilité géographique d'un cadre}

Ainsi que l'illustre la trajectoire de l'actuel responsable du service informatique, les agents recrutés au cours de la dernière décennie ont souvent connu plusieurs formes de mobilité. Après un bac $\mathrm{C}$ et un DUT en informatique, cet homme a brièvement travaillé dans deux entreprises d'informatique du chef-lieu départemental. En 1984, il réussit le concours de programmeur organisé par sa mairie, où il travaille jusqu'en 1997, gravissant progressivement les échelons : technicien chef par examen professionnel en 1993 (qui le conduit à changer de service), ingénieur subdivisionnaire sur concours en 1995. En 1997, il répond à une annonce de la ville de Tozay, parue dans un quotidien régional, et devient responsable du service informatique. Sa mobilité spatiale (partielle, puisqu'il habite toujours au chef-lieu) correspond également à une progression professionnelle (il prend la direction d'un service) et à une promotion hiérarchique (comme ingénieur chef).

Ce renouvellement de l'encadrement doit être rapporté à la strate démographique à laquelle Tozay appartient. C'est dans les villes de 10000 à 40000 habitants que le taux de rotation des DGS a été le plus important au cours des quinze dernières années (Le SAOut, 2011, p. 8). Le cas tozayien suggère qu'il pourrait en être de même pour les cadres moins haut placés. En effet, dans les collectivités de cette taille, les places offertes au sein de l'administration communale, qui peuvent être satisfaisantes pour les agents d'exécution ( $c f$. supra), ne le seraient plus pour les cadres. Face à un nombre réduit de postes accessibles, plusieurs cas de départ de cadres tozayiens vers d'autres collectivités nous ont été rapportés. Des désaccords quant aux projets d'aménagement urbain ont été les moteurs de plusieurs de ces mutations, au début des années 1990, témoignant de l'importance de la politisation fonctionnelle de l'encadrement territorial (Dion, 1986). C'est d'ailleurs en 2001 qu'est recruté le premier directeur général des services issu de la mobilité géographique plutôt que de la promotion interne. Son recrutement à l'issue d'un parcours ascendant dans plusieurs villes politiquement proches de Tozay illustre le maintien du repérage politique - et plus exactement mayoral - de l'encadrement. Or celui-ci ne s'inscrit plus dans le cadre local des réseaux associatifs, syndicaux ou partisans mais dans l'espace national bipolarisé (gauche/droite) de circulation des cadres territoriaux. Les préoccupations politiques rejoignent ici des visées pragmatiques. Le changement d'employeur est souvent synonyme d'augmentation de salaire et les mobilités vers le privé, auxquelles cette étude ne donne pas accès, sont les plus payantes (KouBI, 2005, p. 80).

Bien que polarisé entre les filières technique et administrative (regroupant respectivement $60 \%$ et $20 \%$ des effectifs à Tozay), l'emploi public local ne s'y résume toutefois pas. Les agents des filières culture, animation et sports (18\% des effectifs dans cette ville $\left.{ }^{(26)}\right)$ se distinguent nettement des «administratifs » et des «techniques». L'institutionnalisation plus tardive de ces filières au sein de la FPT explique que ces agents aient été plus souvent contractuels, parfois pendant de longues années ${ }^{(27)}$. De manière complémentaire, la nature de leurs activités (souvent à temps partiel, parfois saisonnière) et leur rapport, mi-professionnel, mi-vocationnel, à leur spécialité les tiennent souvent à distance, objective mais aussi subjective, du statut de la fonction publique.

\section{Emploi culturel : un accès tardif au statut}

La trajectoire du professeur de saxophone de la ville correspond bien à ce profil. Né en 1969 à Tozay (où il réside toujours), il commence à enseigner pour la mairie à 21 ans, après avoir fait l'école de musique du chef-lieu. Un peu plus d'un an plus tard, il obtient un temps complet ( 20 heures par semaine au lieu de 9), mais reste contractuel. Au total, dix contrats sont signés entre 1990 et 2002. En 1994, le maire lui écrit un courrier lui demandant de passer le concours pour être titularisé et "s'investir à plus long terme dans [sa] ville». En 1996, il échoue au concours réservé mis en place par le dispositif Perben de résorption de l'emploi précaire. II lui faut attendre 2002 pour devenir assistant d'enseignement artistique (catégorie B), au titre d'un autre dispositif de résorption de l'emploi précaire, qui le dispense de concours.

(26) Ce taux, assez élevé, s'explique par le caractère touristique de la ville et par le fort investissement du maire au pouvoir entre 1971 et 2005 en faveur de ces secteurs. En revanche, il n'y a pas de police municipale et les «médicaux-sociaux» y sont peu nombreux (on les retrouve surtout dans les conseils généraux).

(27) À Tozay, sept des neuf «contractuels permanents» sont professeurs de musique ou d'arts plastiques. 
Cette enquête localisée a permis d'affiner les analyses forgées à partir de l'évolution des normes juridiques et des représentations associées à la fonction publique territoriale. Le constat d'une mobilité professionnelle, en termes de fonctions et de grades, désormais de plus en plus conditionnée par la mobilité géographique atteste de la construction progressive d'un marché interne correspondant à l'ensemble de la FPT et où les agents partagent non seulement un statut, mais aussi des pratiques de gestion - pensons à la progressive généralisation des fiches de poste et des entretiens d'évaluation (GuÉrARD, 2008) - et des définitions communes de l'aptitude professionnelle. À cette évolution des représentations du «bon» agent territorial s'ajoute le relâchement de la régulation statutaire, qui conduit en particulier à embaucher davantage de contractuels. Après avoir diminué suite à la loi statuaire de 1984, la part de non-titulaires a augmenté continûment de 1998 à $2008^{(28)}$. Dans ces cas, la mobilité volontaire vantée par les responsables territoriaux est régulièrement supplantée par une mobilité contrainte par les fins de CDD.

Ces tendances sans doute communes à l'ensemble de la FPT concourent à l'homogénéisation contemporaine de l'action publique locale (ARNAUD, LE BART, PASQUIER, 2006). Toutefois, elles ne sauraient déboucher sur l'unification de ce segment de l'emploi public. Les prérogatives croissantes accordées aux employeurs limitent les convergences entre collectivités. Les moyens et priorités financières (en faveur ou en défaveur des primes par exemple) nourrissent les inégalités salariales entre collectivités. Les mutations sont quant à elles structurées par des logiques d'appariement - entre collectivités reliées par les réseaux de transport, entre collectivités du même bord politique etc. - qui inviteraient à penser plus finement les liens faibles délocalisés qui se substituent progressivement aux liens forts et locaux. Ainsi, la petite ville de Tozay, située en province, au centre d'une région rurale, peine à faire venir à elle les jeunes diplômés de troisième cycle, que l'on trouve nombreux dans les collectivités de petite couronne parisienne, à proximité du cœur universitaire du pays. En revanche, elle parvient à attirer des cadres franciliens en deuxième partie de carrière, attirés par son cadre de vie et par la possibilité d'y exercer des responsabilités ailleurs réservées à des personnes plus gradées qu'eux.

La propension à la mobilité dépend ainsi de la région d'origine et de travail. Connue pour être un

(28) On remarque toutefois une légère diminution de ce taux en 2009, qui se situe autour de $20 \%$ des effectifs (DGAFP, 2011, pp. 82-83). pôle d'attraction pour les fonctionnaires de l'État (Pauron, 2003, p. 199), la région Île-de-France est l'espace par excellence de mise en circulation des cadres territoriaux. Plaçant souvent en tête le conseil régional, une hiérarchie des employeurs se dessine d'ailleurs, selon le régime indemnitaire qu'ils octroient et les perspectives de carrière qu'ils promettent. C'est également en région parisienne que l'on repère les plus fortes aspirations à la mobilité parmi les agents d'exécution. Dans ce contexte suburbain, les conditions de logement et de vie quotidienne accentuent des aspirations à la mobilité de longue date liées au projet de retour dans la région d'origine. La volonté de quitter les communes les plus populaires motive ainsi certains projets de mutation. L'éloignement - professionnel et résidentiel - des grands ensembles participe alors de la prise de distance à l'égard des populations précarisées. Alors que la thématique du rapprochement des administrations vis-à-vis de leurs usagers inspire de nombreuses réformes, l'affaiblissement des liens de familiarité entre habitants et fonctionnaires - pour des raisons sociales au moins autant que géographiques (SiвLOT, 2006) - contribue à transformer les relations de service, dans le sens d'une formalisation voire d'une managérialisation croissante. Par contraste, l'enquête dans la petite ville provinciale de Tozay a montré qu'en dépit des injonctions contemporaines à la mobilité géographique, une large partie des fonctionnaires territoriaux demeurent fortement ancrés dans la collectivité pour laquelle ils travaillent et où ils résident souvent. La banalisation de la mobilité chez les cadres contraste ainsi avec son caractère limité chez les agents de catégorie C. Entre l'encadrement venu d'ailleurs et la majorité d'agents d'exécution ayant peu de perspective de mobilité, les tensions étaient palpables au cours de notre recherche à Tozay (BILAND, 2010b, pp. 137-138). Les premiers semblent avoir intégré la nécessité de «muter pour réussir» et anticipent le risque de désaveu politique, quand le changement d'employeur apparaît coûteux, tout autant que peu probable, aux seconds. La relative substituabilité de leurs compétences professionnelles, ainsi que la difficulté à reconstruire ailleurs leurs arrangements familiaux, fait douter les «catégorie $\mathrm{C}$ » de leur employabilité hors des lieux où se déploie leur modeste capital social.

Pour prendre en considération les traits structurants des carrières tout autant que les significations conférées aux mobilités par les fonctionnaires, et pour appréhender leurs incidences croisées sur le travail des agents et l'action publique locale, le protocole de cette recherche s'est révélé fécond. Le va-et-vient entre les agents et leurs dossiers a permis d'inscrire les expériences individuelles dans leur espace social de possibilité (reconstruit ici sous la forme de trajectoires typiques). Il a mis en lumière les ressorts de mobilités objectivées par l'exploitation quantitative des données administratives. Ces 
différentes approches des mobilités ne convergent pas toujours. Ainsi, la comparaison «toutes choses égales par ailleurs» des mobilités des hommes et des femmes débouche sur des variations limitées, tandis que celles-ci s'élargissent quand on prend en compte la structure genrée des parcours professionnels (SILVERA, 1996) ou encore les rapports, différenciés selon le genre, à la carrière (TESTENOIRE, 2001). En somme, en étudiant les appropriations locales (par les employeurs publics) et sociales (par les fonctionnaires) de l'injonction à la mobilité, cet article confirme la portée heuristique des regards croisés sur les mobilités professionnelles.

\section{Bibliographie}

Arnaud L., Le Bart C., Pasquier R. (dir.) (2006), Idéologies et action publique territoriale. La politique change-t-elle encore les politiques?, Presses Universitaires de Rennes.

Auby J-M., Auby J-B., Didier J-P. (2005), Droit de la fonction publique, Dalloz.

BACHelet F. (2006), «Sociologie, formation et carrière des hauts fonctionnaires territoriaux", Annuaire des Collectivités locales, CNRS éditions, pp. 99-103.

Becker H. (1970), Sociological work. Method and Substance, Aldine.

Bellanger, E. (2004), Administrer la banlieue municipale. Activité municipale, intercommunalité, pouvoir mayoral, personnel communal et tutelle préfectorale en Seine banlieue des années 1880 aux années 1950, thèse d'histoire, Université Paris 8.

Bellanger E. (2001), «L'école nationale d'administration municipale. Des «sans-grade» devenus secrétaires généraux », Politix, no 53, pp. 145-171.

Bellanger E. (2008), «Le personnel communal ou l'empreinte du territoire : construction d'un groupe, politisation et sociabilité municipale», in Bellanger E., Girault J. (dir.), Villes de banlieue. Personnel communal, élus locaux et politiques urbaines en banlieue parisienne au 20e siècle, Créaphis, pp. 151-189.

BILAND É. (2009), ««Moderniser les ressources humaines» dans une petite ville française : appropriations et contournements des normes juridiques et gestionnaires », Pyramides, no 17, pp. 15-34.

BiLAND É. (2010a), «Carrières concourantes. Éléments sur la socialisation par les concours administratifs territoriaux », Terrains et Travaux, no 17, pp. 57-73.

BILAND É. (2010b), «Les usages du statut par les employeurs publics locaux : embaucher et gérer les carrières à la ville des années 1970 aux années 2000 », in Cartier M., Retière J-N., Siblot Y. (dir.), Les salariés à statut. Genèses et cultures, Presses Universitaires de Rennes, pp. 127-139.

Blum A. de la Gorce G., Thélot C. (1985), «Mobilité sociale et migration géographique», Population, v. 40, no 3 , pp. 405.
Boltanski L., Chiapello غ̀ (1999), Le nouvel esprit du capitalisme, Gallimard.

Bozon M., HÉrAn F. (2006), La formation du couple. Textes essentiels pour la sociologie de la famille, La Découverte.

Burlen K., Thoenig J-C. (1998), «Les secrétaires généraux des villes », Politiques et management public, v. 16, no 1 , pp. 141-172.

Castel R. (2003), L'insécurité sociale. Qu'est-ce qu'être protégé?, Seuil.

Chapoulie S. (2000), «Une nouvelle carte de la mobilité professionnelle», Économie et statistique, no 331 , pp. 25-45.

Chevallier G. (1934), Clochemerle, Le livre de poche, réédition 2004.

Delvové P. (2006), «La privatisation du service de l'État», Pouvoirs, no 117, pp. 107-120.

Dgafp (2010), Rapport annuel sur l'état de la fonction publique 2009-2010, La Documentation française.

DGAFp (2011), Rapport annuel sur l'état de la fonction publique 2010-2011, La Documentation française.

DGCL (2011), Les collectivités locales en chiffres 2011, ministère de l'Intérieur.

Dion S. (1986), La politisation des mairies, Economica.

Doeringer P., Piore M. (1971), Internal Labor Markets and Manpower Analysis, Heath Lexington Books.

Dupays S. (2003), «La mobilité dans la fonction publique : mythe ou réalité?», in Observatoire de l'emploi public, Rapport annuel 2003, annexe 3, La Documentation française, pp. 39-45.

DuRAND D. (2004), Une histoire de la fonction publique territoriale, La Dispute.

Fontaine J. (1986), «Être cadre de catégorie A dans la mairie d'une grande ville», Politiques et management public, v. 4, no 3, pp. 23-62.

Gollac S. (2005), «La Fonction Publique : une voie de promotion sociale pour les enfants des classes populaires? Une exploitation de l'enquête emploi $2001 »$, Sociétés Contemporaines, $\mathrm{n}^{\circ}$ 58, pp. 41-63. 
GuÉrARD S. (dir.) (2008), La GRH publique en questions : une perspective internationale, L'Harmattan.

Koubi M. (2005), «La mobilité professionnelle dans les collectivités territoriales. Une première évaluation», in Observatoire de l'emploi public, Rapport annuel 20042005, Annexes, pp. 61-81.

LE BART C. (2011), «Le marketing territorial», in Cole A., Guigner S., Pasenier R. (dir.), Dictionnaire des plitiques teritoriales, Presses de Sciences Po, Paris.

LE LideC P. (2006), «Choix des modes de gestion des services publics et politiques de recrutement des communes. Quelle place pour la variable partisane?», in Arnaud L., Le Bart C., Pasquier R. (dir.), Idéologies et action publique territoriale. La politique change-t-elle encore les politiques?, Presses Universitaires de Rennes, pp. 35-49.

Le Lidec P., Montricher N. (2004), Décentraliser et gérer. Analyse rétrospective et prospective de l'emploi public dans les collectivités territoriales, La Documentation française.

Le Saout R. (2011), «La mobilité des directeurs généraux des services municipaux comme indicateur d'un rapport renouvelé au travail politique», $11^{\mathrm{e}}$ congrès de l'Association Française de Science Politique, ST 40.

LORRAIN D. (1977), «La situation du personnel communal en France», in IFSA, L'administration des grandes villes, cahiers no 14 , Cujas, Paris.
LORRAIN D. (1991), «De l'administration républicaine au gouvernement urbain», Sociologie du travail, $\mathrm{n}^{\circ} 4$, pp. 461-484.

OBSERVATOIRE DE L'EMPLOI, DES MÉTIERS ET DES COMPÉTENCES (2010), «Les mouvements de personnels territoriaux en 2007», Synthèse, CNFPT, no 29.

Pauron A. (2003), «La mobilité des agents titulaires de l'État», Économie et statistique, no 369-370, pp. 93-111.

Raynaud P. (2003), «L'emploi public est tiré par la fonction publique territoriale», Économie et statistique, no 369-370, pp. 75-92.

RetiÈRe J-N. (2003), «Autour de l'autochtonie. Réflexion sur la notion de capital social populaire», Politix, no 63, pp. 121-143.

Schrameck O. (1995), La fonction publique territoriale, Dalloz, coll. «Connaissance du droit. Droit public», Paris.

Schwartz O. (1998), «La notion de « classes populaires » », mémoire pour l'habilitation à diriger des recherches en sociologie, université de Versailles-Saint-Quentin.

Siblot Y. (2006), Faire valoir ses droits au quotidien. Les services publics dans les quartiers populaires, Presses de Sciences Po.

Silvera R. (1996), «Les discriminations salariales entre hommes et femmes, toutes choses inégales par ailleurs », Economies et sociétés, v. 30, no 11-12, pp. 199-216.

TESTENOIRE A. (2001), «Les carrières féminines : contingence ou projet?», Travail, genre et société, $\mathrm{n}^{\circ} 5$, pp. 117-134. 